\title{
ELECTRONIC STRUCTURE AND MAGNETIC PROPERTIES OF $\mathrm{TiMn}_{3} \mathrm{~N}, \mathrm{TiMn}_{3}$ AND MnTi $\mathrm{M}_{3}$ COMPOUNDS USING TB-LMTO METHOD
}

\author{
V. Sathana ${ }^{1}$, G. Meenakshi' ${ }^{2}$ V. Jayalakshmi ${ }^{3}$ \\ ${ }^{I}$ Research Scholar, Manonmaniam Sundaranar University, Tirunelveli, India, \\ Department of Physics, St. Joseph's College of Arts \& Science (AUTONOMOUS), Cuddalore, India \\ ${ }^{2}$ Department of Physics, KMCPGS, Puducherry \\ ${ }^{3}$ Department of Physics, SRM University, Ramapuram, Chennai, India
}

\begin{abstract}
Tight Binding Linear Muffin Tin Orbital Method has been employed to obtain the electronic and Magnetic properties of TiMn ${ }_{3} N$ and ordered $\mathrm{TiMn}_{3}$ Compounds. The Equilibrium volume has been found for each compound using self-consistent band structure calculations at several lattice parameters. Obtained results reveals that TiMn 3 is ferromagnetic with $3.3624 \mu B$ as magnetic

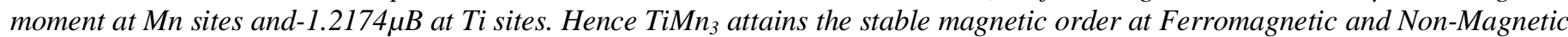
calculations, while TiMn ${ }_{3} N$ shows the nonmagnetic phase as a stable one during FM and NM calculations.
\end{abstract}

Keywords: Ferromagnetic, Non-Magnetic, Tight Binding Linear Muffin Tin Orbital method

\section{INTRODUCTION}

Titanium based Nitrides are of great importance because of its tremendous industrial applications such as Lithium-ion batteries, energy storage, fuel cells and biomedical industries. Various Experimental and Theoretical works has been done in Titanium nitrides that provides ultra-hardness, corrosion resistance and mechanical resistance. Analyzing the magnetic properties of these nitrides offers high wear resistant property to the materials. [1-16]. Some Titanium nitrides are considered as high technology materials often used in microelectronics, space technology, Aero planes industry and biomaterials, due to their exceptional physical and chemical properties [17-19]. These type of compounds are chemically stable and shows good corrosion resistance even at room temperature and they are used as biocompatible layers for orthopedic and dental implants. Finally, their hardness is among the highest next to diamond. It has contributed to the industrial use of titanium nitrides, as good candidates for applications needing high wear resistance [20, 21]. On the other hand, titanium nitrides are widely used in semi-conductors technology as dilution barriers [22, 23]. Titanium nitrides doped with transition metal atom displays a wide range of interesting phenomenon of converting the semiconducting materials to magnetic and superconducting compounds [24]. Also it has got excellent electrical and thermal conductivity, high chemical and thermal stability and good wear and corrosion resistance. All these properties make them suitable for many technological applications. Titanium nitride is also used as an electrically conducting barrier. Thus these compounds have great scientific and technological interest [25]. The investigated structural properties of the $\mathrm{XFe}_{3} \mathrm{~N}$ cubic ternary iron based nitrides reveals that in the cases of $\mathrm{ScFe}_{3} \mathrm{~N}, \mathrm{CoFe}_{3} \mathrm{~N}$,
$\mathrm{NiFe}_{3} \mathrm{~N}, \quad \mathrm{CuFe}_{3} \mathrm{~N}$ and $\mathrm{ZnFe}_{3} \mathrm{~N}$, the equilibrium configurations corresponds to the Pm-3m structure [27]. Substitution of $\mathrm{Zn}$ for $\mathrm{Fe}$ in $\gamma^{\prime}-\mathrm{Fe}_{4} \mathrm{~N}$ improves the corrosion resistance as well as mechanical properties [26]. The electronic structure of compounds $\mathrm{FeV}_{3}, \mathrm{VFe}_{3}, \mathrm{~V}_{4} \mathrm{~N}, \mathrm{VFe}_{3} \mathrm{~N}$, and $\mathrm{FeV}_{3} \mathrm{~N}$, has been studied using the LMTO method. With the use of total energy calculations the equilibrium lattice parameters for these compounds are calculated. Since the $\mathrm{V}_{4} \mathrm{~N}, \mathrm{FeV}_{3} \mathrm{~N}$ and $\mathrm{VFe}_{3} \mathrm{~N}$ nitrides shows nonmagnetic, the substitution of iron by vanadium atoms destroys the ferromagnetic order of the- $\mathrm{Fe}_{4} \mathrm{~N}$ nitride [28]. Magnetic and electronic properties of the two compounds $\gamma^{\prime}-\mathrm{AgFe}_{3} \mathrm{~N}$ and $\gamma^{\prime}-\mathrm{AuFe}_{3} \mathrm{~N}$ has been studied using LMTO calculations and correlated with experimental results [29]. Similarly, LMTO method are engaged to investigate the electronic structure and local magnetic properties of the ferromagnetic iron nitrides $\mathrm{RuFe}_{3} \mathrm{~N}, \mathrm{ZnFe} \mathrm{H}_{3} \mathrm{~N}, \mathrm{InFe}_{3} \mathrm{~N}, \mathrm{Fe}_{3} \mathrm{BN}, \mathrm{PdFe}_{3} \mathrm{~N}, \mathrm{MnFe}_{3} \mathrm{~N}$ and $\mathrm{SnFe}_{3} \mathrm{~N}[30-35]$.

Considering all the above references, $\gamma^{\prime}-\mathrm{Fe}_{4} \mathrm{~N}$ has been used as model compound substituting the transition metal atoms in place of $\mathrm{Fe}$ atom and the electronic and various other properties of these compounds has been studied using LMTO methods of calculation. In the present work, three Fe atoms are replaced by $\mathrm{Ti}$ atom and one $\mathrm{Fe}$ atom by other transition metal atom $\mathrm{Mn}$ to analyze the magnetic properties of those compounds with and without nitrogen.

\section{METHODS OF CALCULATION}

Basic research in the material science includes the study of electronic structure as the beginning for all the physical properties. Band structure calculations have proved to be tremendously useful in relating the vital feature of all band 
calculations with the large experimental data. Band structure calculations may be referred as linear method, introduced by O. k. Anderson in 1971, which are later derived as many efficient computational patterns. One of these linear methods that solves self-consistent electronic structure problem in an extremely effective way is the Linear Muffin Tin Orbital (LMTO) method. Using this method various calculations for infinite crystals and ground state properties of real materials may be estimated [36]. The simplest and most widely used one-electron Hamiltonian is tight-binding (TB) method with minimal base and in its two-Centre approximation has been necessary for self-consistent density-functional calculations. The transformation of muffin-tin orbitals into a TB item frequently and successfully used in first principle band structure calculations. The transformation made by LMTO method is exact for all results obtained. By using this method or with the almost identical augmented spherical wave method, the results can always be reproduced by the TB method. The TB-LMTO's have an almost universal decay with the socalled atomic sphere approximation $[37,38]$.

The electronic structure and ground state properties of $\mathrm{TiMn}_{3} \mathrm{~N}, \mathrm{TiMn}_{3}$ and $\mathrm{MnTi}_{3}$ compounds have been studied by using self-consistent TB-LMTO method. These compounds crystallize the simple cubic structure with space group Pm-3m (space group no: 221). In order to find the phase stability of the compounds, the total energies has been calculated for these compounds and fitted with Birch Murnaghan equation of state [39-40]. The ferromagnetic and nonmagnetic calculations has also been performed to investigate the magnetic behavior of the compounds.
It is assumed that the crystal structures of titanium based manganese nitride and titanium nitride as simple cubic, in which the metal atoms occupy the corner sites and the face centered position, while nitrogen atoms occupy the bodycentered sites. A spin-polarized LMTO calculation has been performed using the Von Barth and Hedin Parameterization [41] for exchange-correlation energy of the electron gas.

Progressing LMTO calculations has been made without spin-orbit interaction but including the mass correction terms. For $\mathrm{TiMn}_{3} \mathrm{~N}$ nitrides the Wigner-Seitz Spheres ( $\mathrm{Si}$ ) around Titanium and Manganese atoms has been taken to be of equal size. The one-electron potentials has been selfconsistently obtained using reciprocal space sums with 216 $\mathrm{k}$-points having 20 irreducible $\mathrm{k}$ points within the entire part of the Brillouin zone. The self-consistent iterations has been carried out until energy convergence on a scale better than $0.1 \mathrm{mRy}$. Solutions for the Schrodinger equations has been used s, p, d, f basis functions for metals (Mn, Ti) and s, p, d basis functions for nitrogen. The densities of states (DOS) has been calculated as a sum of delta functions for $6 \times 6 \times 6$ mesh points. In the present study, $3 d$ and 4 s orbital's of $\mathrm{Ti}$, $3 \mathrm{~d}$ and $4 \mathrm{~s}$ orbital's of $\mathrm{Mn}$ and $2 \mathrm{~s}$ and $2 \mathrm{p}$ orbitals of $\mathrm{N}$ has been treated as valence states.

\section{RESULTS AND DISCUSSION}

The Total energy calculation has been performed for compounds without nitrogen, namely, $\mathrm{TiMn}_{3}$ and $\mathrm{MnTi}_{3}$. The calculated binding curves between total energies in Rydberg and lattice parameters in atomic units of those two compounds have been shown in Fig 1.

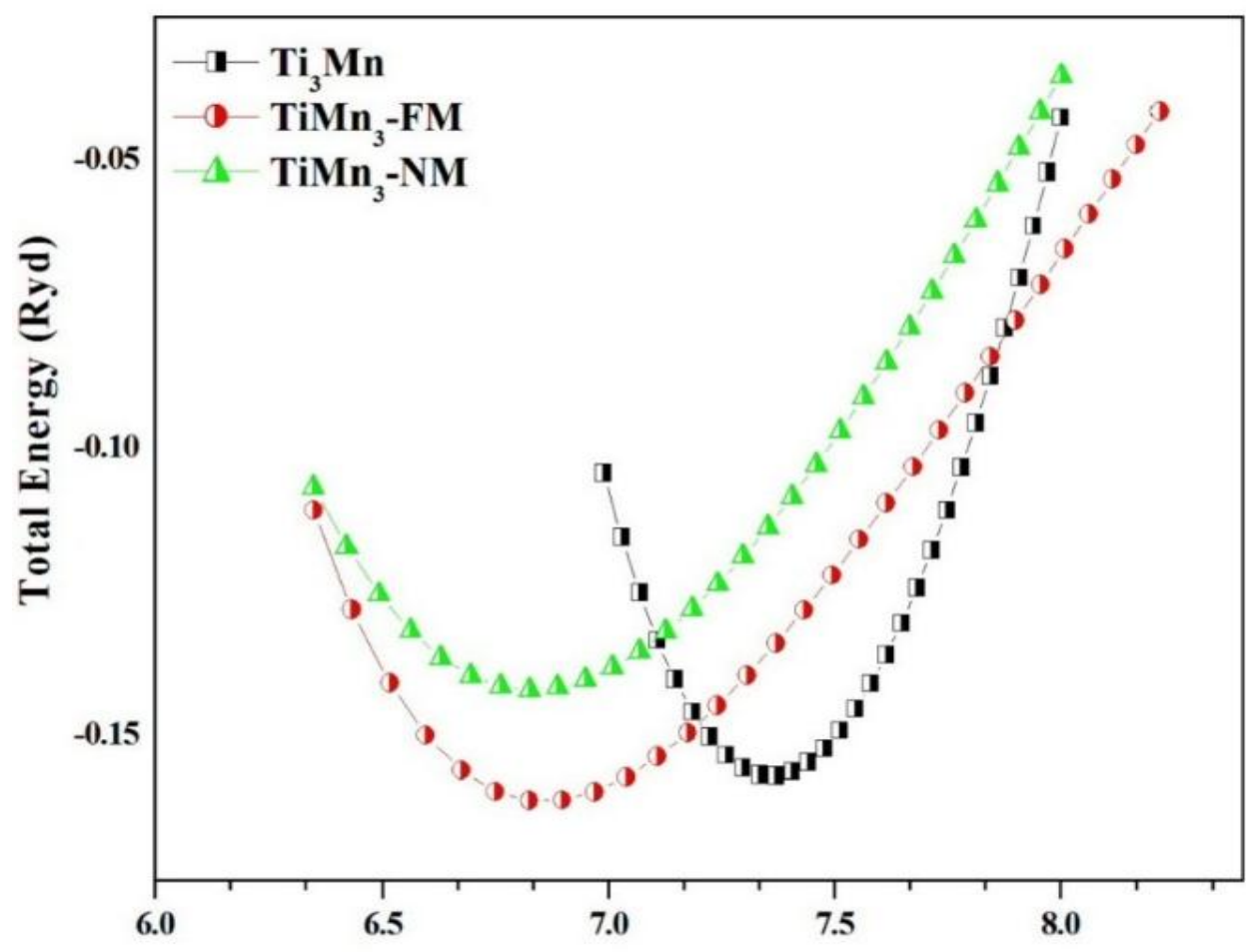

Lattice Parameter (a.u.)

Fig.1. Binding curves between total energies (in Rydberg) versus lattice spacing ' $a$ ' (atomic units) for the compounds $\operatorname{TiMn}_{3}(\mathrm{NM}$ $\& \mathrm{FM})$ and $\mathrm{MnTi}_{3}$. 
Table.1. Estimated lattice parameters and Bulk moduli for the compounds $\mathrm{Ti}_{4} \mathrm{~N}, \mathrm{TiMn}_{3} \mathrm{~N}, \mathrm{TiMn}_{3}-\mathrm{FM}, \mathrm{TiMn}_{3}-\mathrm{NM}$ and $\mathrm{MnTi}{ }_{3}$

\begin{tabular}{|l|l|l|l|l|l|}
\hline & $\mathrm{Ti}_{4} \mathrm{~N}$ & $\mathrm{TiMn}_{3} \mathrm{~N}$ & $\mathrm{TiMn}_{3}(\mathrm{FM})$ & $\mathrm{TiMn}_{3}(\mathrm{NM})$ & $\mathrm{MnTi}_{3}$ \\
\hline A (a.u) & 7.8265 & 7.2139 & 7.8729 & 6.8299 & 7.3585 \\
\hline B (GPa) & 192.27 & 388.28 & 249.85 & 247.47 & 154.78 \\
\hline
\end{tabular}

A drastic change in the lattice spacing has been observed from $\mathrm{MnTi}_{3}$ to $\mathrm{TiMn}_{3} \mathrm{~N}$ while including Nitrogen as shown in Table.1. Titanium is at face-centered positions for $\mathrm{MnTi}_{3}$ and a ferromagnetic calculation gives null local magnetic moments at $\mathrm{Mn}$ and $\mathrm{Ti}$ sites. It denotes that the stable phase of $\mathrm{MnTi}_{3}$ is nonmagnetic, has been evidenced by analyzing the occupation numbers for this compound. Also it shows a charge transfer from Ti atoms to Mn atoms that populate the spin-down d-states giving an equal occupation numbers for both spin directions.Figure.1. shows that $\mathrm{TiMn}_{3}$ attains two binding curves through nonmagnetic (NM) and ferromagnetic (FM) calculations. Regarding the analysis of the occupation numbers, Mn atoms receives the net magnetic moment from spin-down d-electrons that has been partially unoccupied whereas Ti atoms occupies spin-up d-states, that results in the opposite local magnetic moments at $\mathrm{Fe}$ and $\mathrm{Ti}$ sites at theoretical equilibrium volume. The magnetic moment vanishes for lower volumes (at high pressure) as shown in fig. 2.

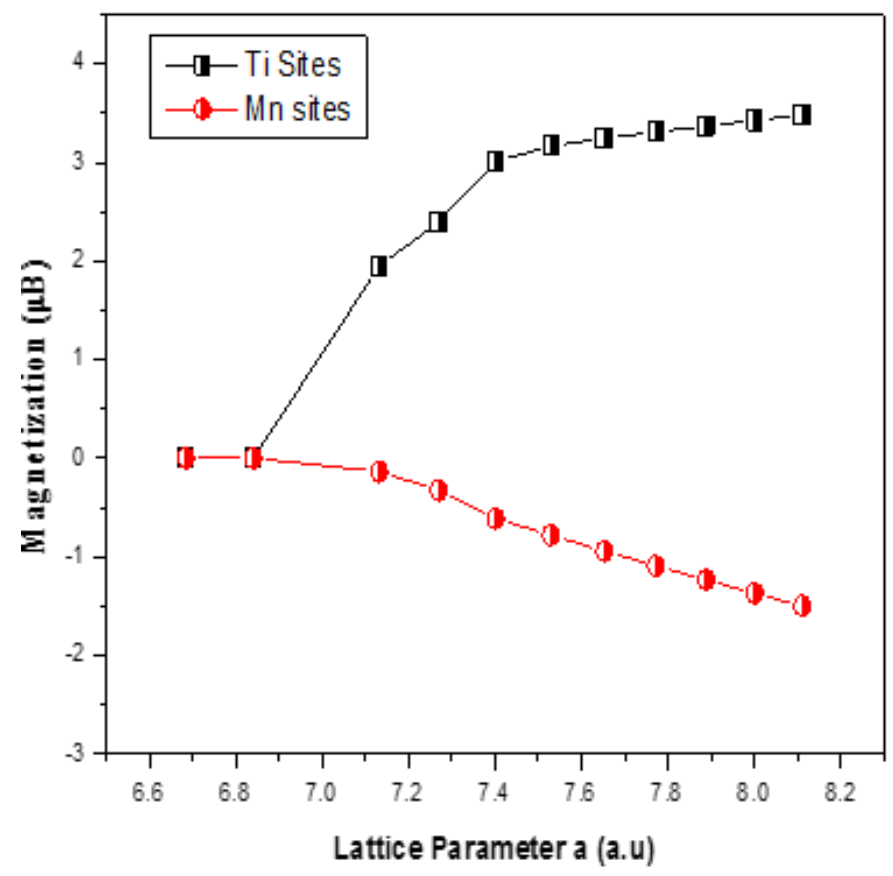

Fig.2 Magnetization (in Bohr Magnetons) versus Lattice Parameter (in atomic units) for $\mathrm{TiMn}_{3}$

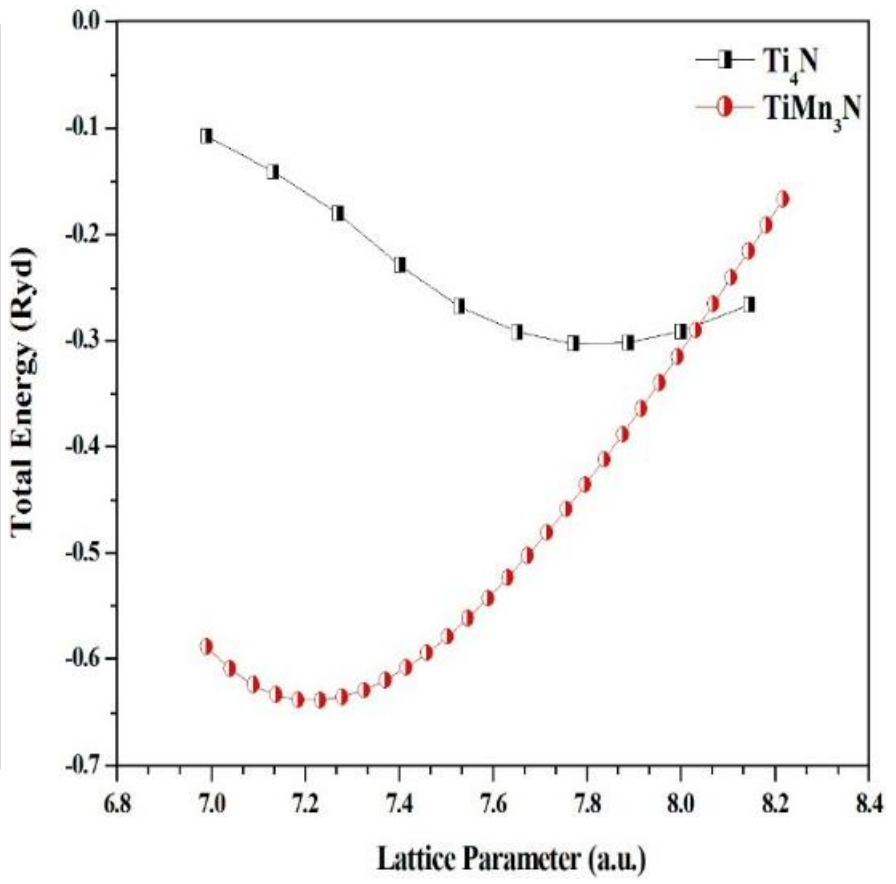

Fig.3 Total Energy (in Rydberg) versus Lattice parameter (in atomic units) for $\mathrm{Ti}_{4} \mathrm{~N}$ and $\mathrm{TiMn}_{3} \mathrm{~N}$

The above two branches NM and FM states in figure.2. overlaps at a junction, where moment can be zero without any change in total energy of the system presents the first order magnetic shift. Figure.3. provides the binding curves for $\mathrm{Ti}_{4} \mathrm{~N}$ and $\mathrm{TiMn}_{3} \mathrm{~N}$ attained through Birch- Murnaghan fitting and the equilibrium lattice parameters found are given in table.1.The electronic distribution and charge transfer of titanium, manganese and nitrogen atoms are calculated using ferromagnetic calculations and given in Table.2.

Table.2. Number of electrons in each state per spin, charge transfer at each site and Fermi energy.

\begin{tabular}{|c|c|c|c|c|c|c|c|c|c|}
\hline \multirow{2}{*}{$\mathrm{TiMn}_{3} \mathrm{~N}$} & \multicolumn{3}{|l|}{$\mathrm{Ti}$} & \multicolumn{3}{|l|}{$\mathrm{Mn}$} & \multicolumn{3}{|l|}{$\mathrm{N}$} \\
\hline & Spin up & Spin down & Difference & Spin up & Spin down & Difference & Spin up & Spin down & Difference \\
\hline $\mathrm{n}_{\mathrm{s}(\text { electrons/spin) }}$ & 0.53189 & 0.53189 & 0.0000 & 0.17025 & 0.17025 & 0.0000 & 0.77676 & 0.77676 & 0.0000 \\
\hline $\mathrm{n}_{\mathrm{d}(\text { electrons/spin) }}$ & 1.90301 & 1.90301 & 0.0000 & 2.60236 & 2.60236 & 0.0000 & 0.06498 & 0.06498 & 0.0000 \\
\hline$\Delta \mathrm{Q}_{\text {(electrons) }}$ & \multicolumn{3}{|c|}{-1.28325} & \multicolumn{3}{|c|}{0.00177} & \multicolumn{3}{|c|}{-2.33075} \\
\hline $\mathrm{E}_{\mathrm{F} \text { (Rydberg) }}$ & \multicolumn{9}{|c|}{-0.121893} \\
\hline
\end{tabular}



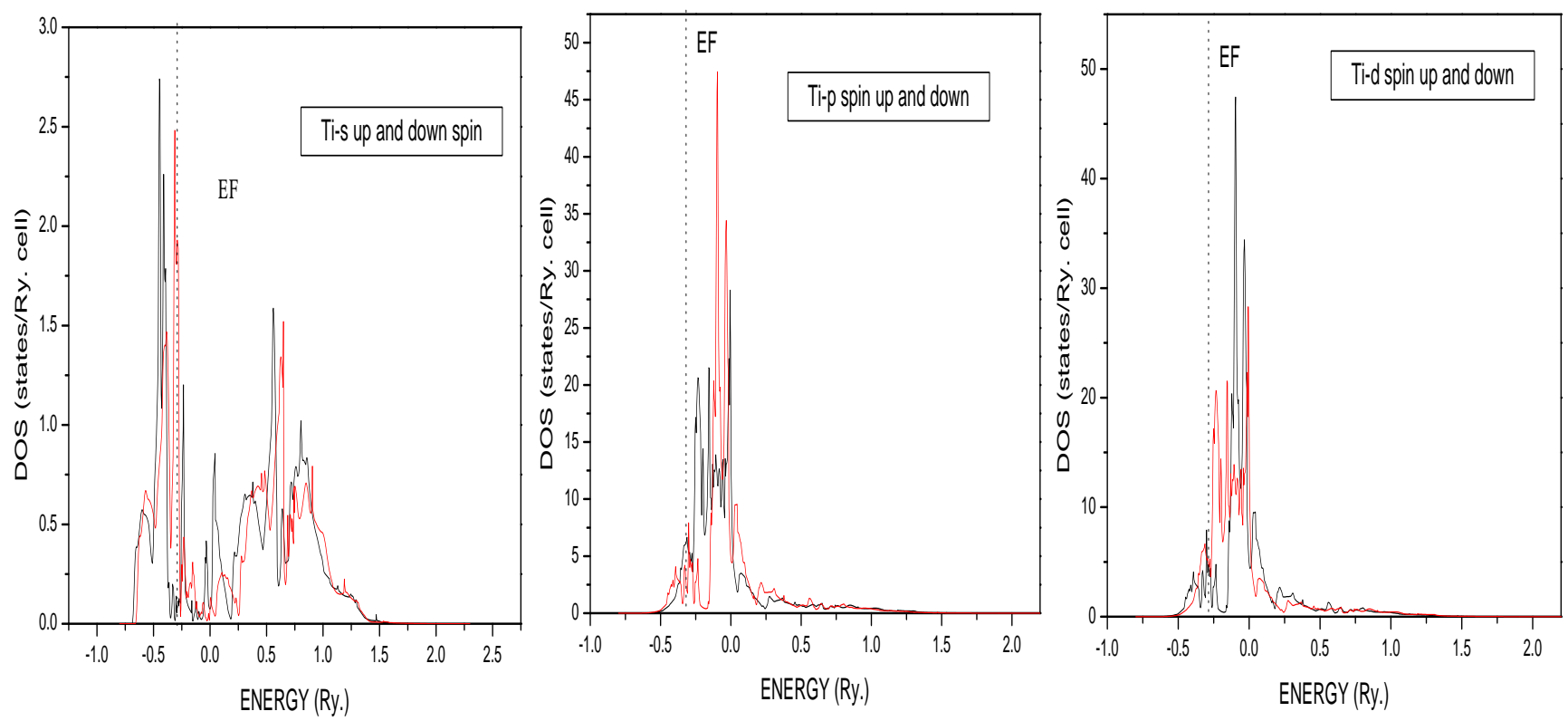

Fig.4 The s, p and d projected densities of states for spin up and spin down electrons at Ti sites for $\mathrm{TiMn}_{3}$.
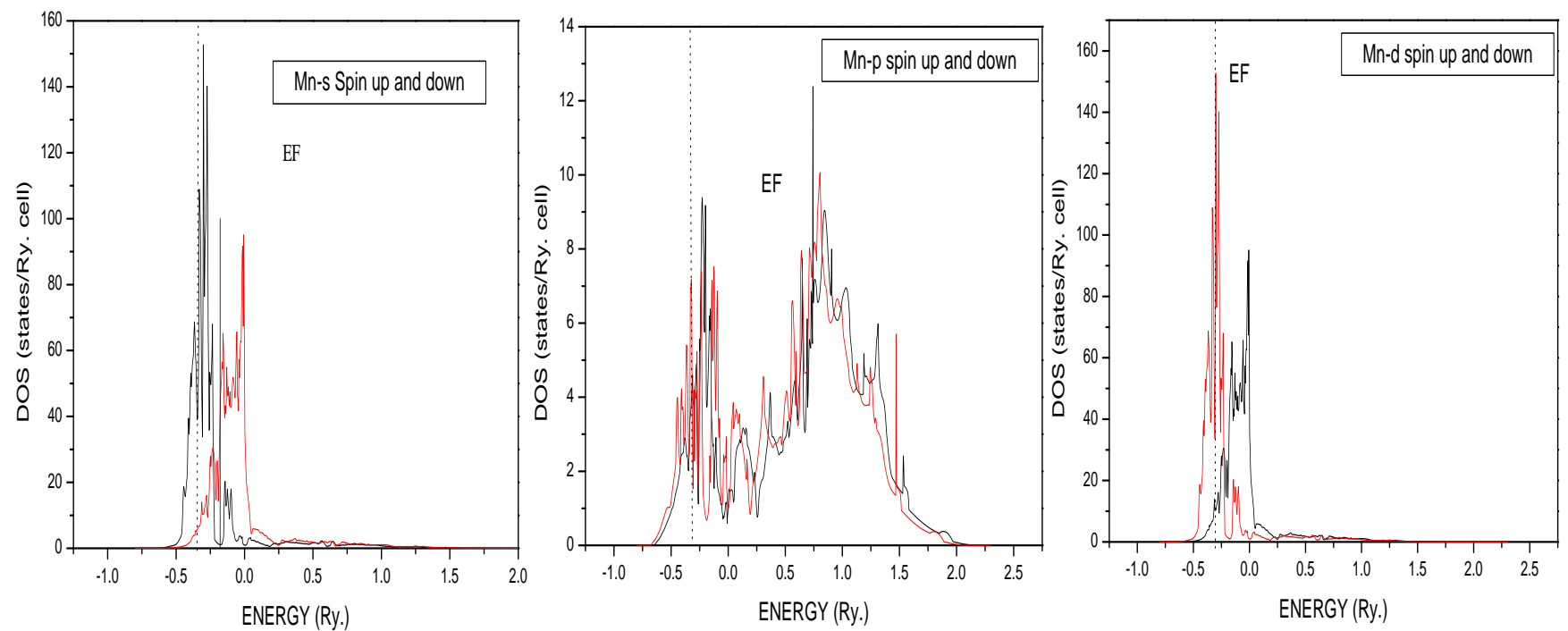

Fig.5 The s, p and d projected densities of states for spin up and spin down electrons at Mn sites for $\mathrm{TiMn}_{3}$.
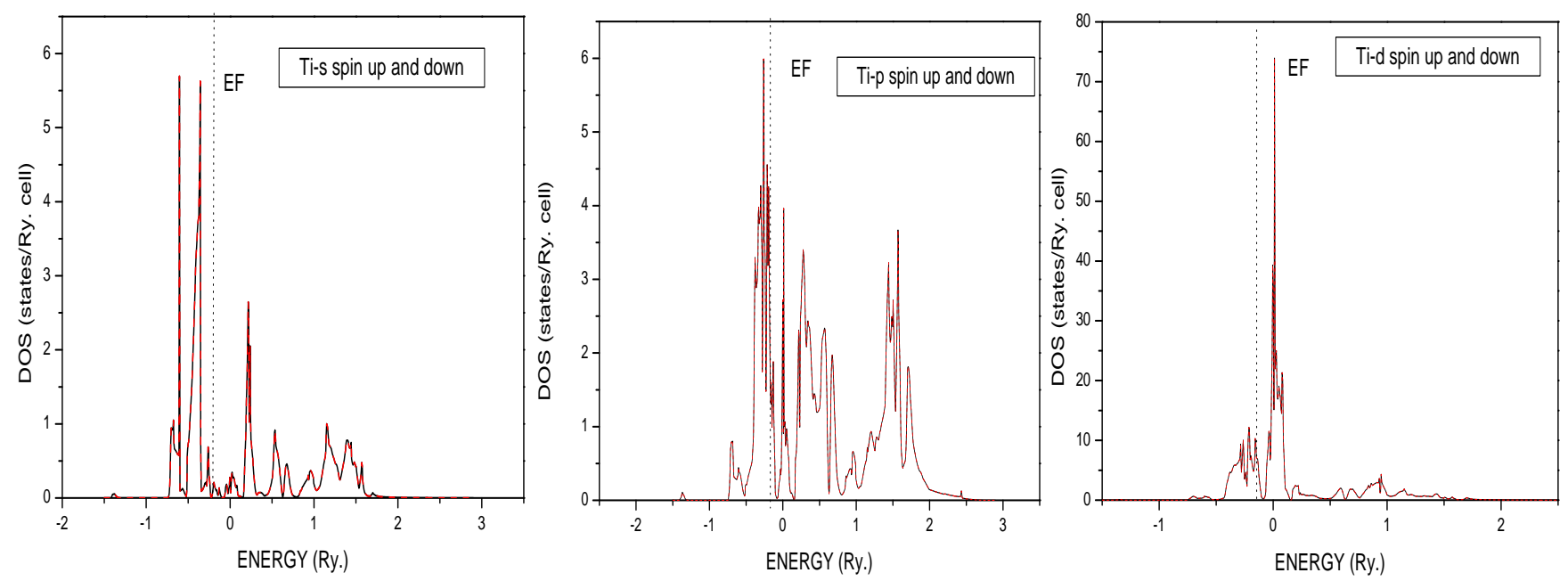

Fig.6 The s, p and d projected densities of states for spin up and spin down electrons at Ti sites for $\mathrm{TiMn}_{3} \mathrm{~N}$. 

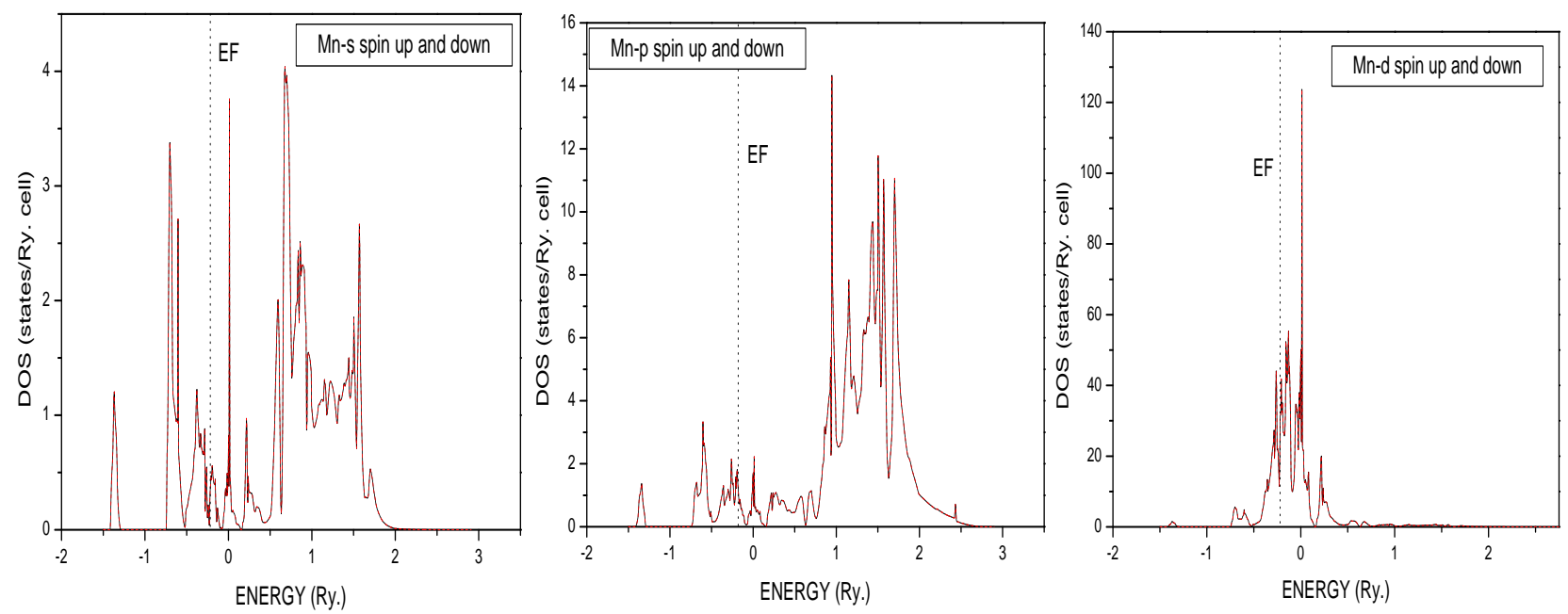

Fig.7 The s, p and d projected densities of states for spin up and spin down electrons at Mn sites for $\operatorname{TiMn}_{3} \mathrm{~N}$
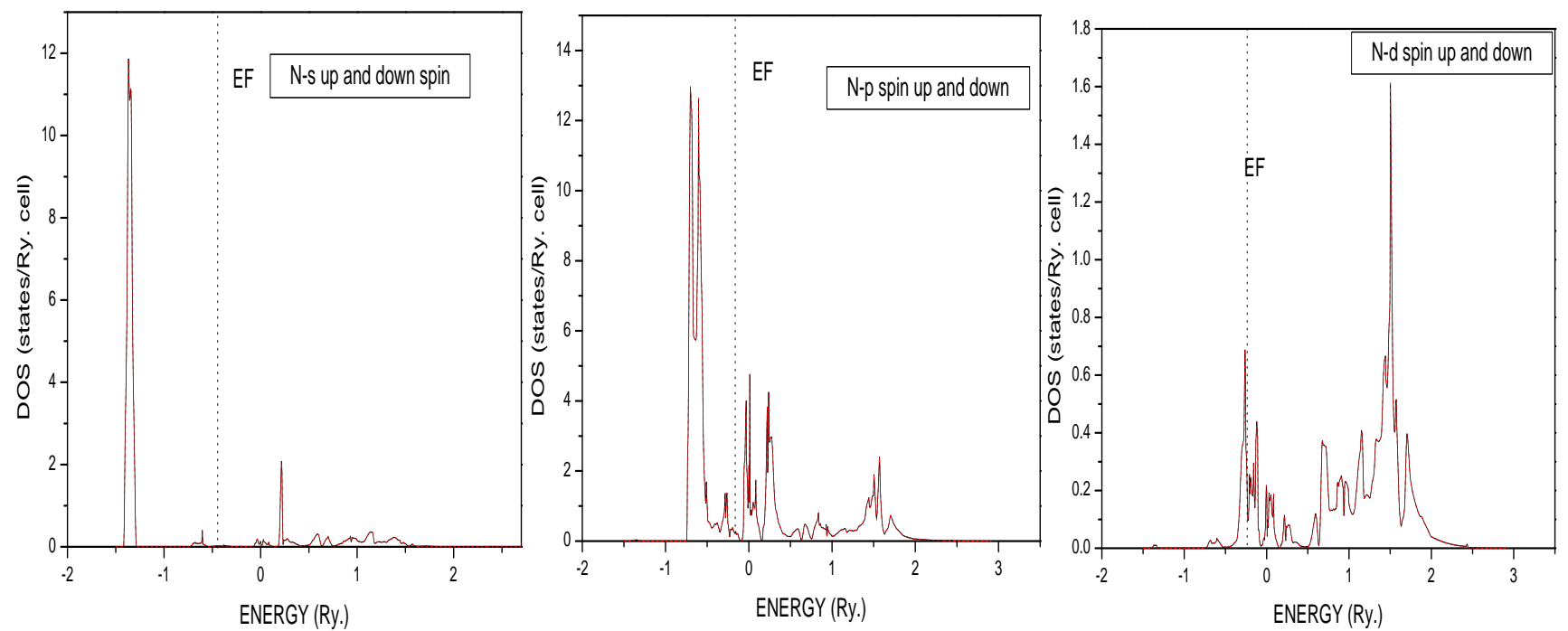

Fig.8 The s, p and d projected densities of states for spin up and spin down electrons at $\mathrm{N}$ sites for $\mathrm{TiMn}_{3} \mathrm{~N}$.

The $l$-projected densities of states ( $l$-DOS) at the theoritical equilibrium volume for both compounds has been investigated to get clear knowledge about the trends of the chemical bonds. Figure 4-8 shows the calculated s,p and d densities of states for both spin directions at Ti and Mn sites for $\mathrm{TiMn}_{3}$ and $\mathrm{Ti}, \mathrm{Mn}$ and $\mathrm{N}$ sites for $\mathrm{TiMn}_{3} \mathrm{~N}$. The potentials obtained for spin up and spin down electrons remains same(in the case of $\mathrm{TiMn}_{3} \mathrm{~N}$ ) will be easily understood for nonmagnetic electronic behavior. Contrarily, ferromagnetic compounds (in the case of $\mathrm{TiMn}_{3}$ ) exhibits the different DOS for up and down spin populated by the unoccupied spin down d-states provides a net local magnetic moment at these sites as shown in figure. 4-5. Hence the increase in bulk moduli according to these interactions of $\mathrm{TiMn}_{3} \mathrm{~N}$ has the higher bulk modulus(table.1.) than others which are reflected in DOS at various sites that has been analyzed clearly. Hence these changes in the densities of states profiles of $\mathrm{TiMn}_{3} \mathrm{~N}$ and $\mathrm{TiMn}_{3}$ is due to changes in the self - consistent potentials felt by the electrons after interfering nitrogen.

\section{CONCLUSION}

The electronic structure, ground state properties and magnetic behavior of compounds $\mathrm{TiMn}_{3} \mathrm{~N}, \mathrm{TiMn}_{3}$ and $\mathrm{MnTi}_{3}$ are well known due to their excellent applications has been analyzed using TB-LMTO method. The total energy calculations have been engaged to determine the stable crystallographic parameters. The ferromagnetic calculations gives a null local magnetic moments for $\mathrm{MnTi}_{3}$ at $\mathrm{Mn}$ and $\mathrm{Ti}$ sites which indicates that the stable phase of $\mathrm{MnTi}_{3}$ is nonmagnetic as a result of charge transfer from titanium atom to Manganese atom giving an equal occupation numbers for both spin up and spin down directions and are verified by projected DOS profiles. Concerning $\mathrm{TiMn}_{3}$, the value of magnetic moments after the analysis of Ferromagnetic calculations are $-1.2174 \mu \mathrm{B}$ at Titanium sites and $3.3624 \mu \mathrm{B}$ at Manganese sites and the total magnetic moment per unit cell $8.8698 \mu \mathrm{B}$. The magnetic moments has been calculated for both sites for $\mathrm{TiMn}_{3}$ as a function of lattice parameter shows that, for larger volume there exists a ferromagnetic order but the null magnetic moment exists with a small decrease in the lattice constant nearly $\mathrm{a}=6.82$ a.u. destroying the ferromagnetic order. On the subject of 
$\mathrm{TiMn}_{3} \mathrm{~N}$ nitride, ferromagnetic calculations gives null magnetic moments at all sites and provides the ground state of this nitride is nonmagnetic. Hence it is concluded that inclusion of nitrogen with $\mathrm{TiMn}_{3}$ destroys the ferromagnetic order due to the charge transfer from $\mathrm{Ti}$ and $\mathrm{N}$ to $\mathrm{Mn}$ atom, provides different spin and these densities of states has also been analyzed for $\mathrm{TiMn}_{3}$ compound.

\section{REFERENCES}

[1] Linfei Lai, Jixin Zhu, Baosheng Li, Yongda Zhen, Zexiang Shen, Qingyu Yan, Jianyi Lin, Electrochimica Acta, Volume 134, 10 July 2014, Pages 28-34.

[2] Gaojie Xu, Lixue Zhang, Chaowei Guo, Lin Gu, Xiaogang Wang, Pengxian Han, Kejun Zhang, Chuanjian Zhang, Guanglei Cui, Electrochimica Acta, Volume 85, 15 December 2012, Pages 345351.

[3] Shanmu Dong, Xiao Chen, Xiaoying Zhang, Guanglei Cui, Coordination Chemistry Reviews, Volume 257, Issues 13-14, July 2013, Pages 19461956.

[4] A.P. Serro, C. Completo, R. Colaço, F. dos Santos, C. Lobato da Silva, J.M.S. Cabral, H. Araújo, E. Pires, B. Saramago, Surface and Coatings Technology, Volume 203, Issue 24, 15 September 2009, Pages 3701-3707.

[5] A. Königer, J.W. Gerlach, H. Wengenmair, C. Hammerl, J. Hartmann, B. Rauschenbach, Surface and Coatings Technology, Volume 84, Issues 1-3, October 1996, Pages 439-442.

[6] P.R. Mezger, N.H.J. Creugers, Journal of Dentistry, Volume 20, Issue 6, December 1992, Pages 342344.

[7] C.A. Kuhnen, A.V. dos Santos, Journal of alloys and compounds, Volume 384, 2004, Pages 80-87.

[8] A.V. dos Santos, M.I. da Costa, C.A. Kuhnen, Journal of Magnetism and Magnetic Materials, Volume 166, Issues 1-2, 1 February 1997, Pages 223-230.

[9] C.A. Kuhnen, E.Z. da Silva, Solid State Communications, Volume 61, Issue 8, February 1987, Pages 475-478.

[10] C.A. Kuhnen, E.Z. da Silva, Journal of Magnetism and Magnetic Materials, Volume 67, Issue 2, June 1987, Pages 260-270.

[11] R.S. de Figueiredo, C.A. Kuhnen, A.V. dos Santos, Journal of Magnetism and Magnetic Materials, Volume 173, Issues 1-2, 1 September 1997, Pages 141-154.

[12] E.Z. da Silva, C.A. Kuhnen, Solid State Communications, Volume 66, Issue 10, June 1988, Pages 1011-1014.

[13] C.A. Kuhnen, A.V. dos Santos, Solid State Communications, Volume 85, Issue 3, January 1993, Pages 273-279.

[14] C.A. Kuhnen, A.V. dos Santos, Journal of Magnetism and Magnetic Materials, Volume 130, Issues 1-3, February 1994, Pages 353-362.
[15] Desheng Xue, Fashen Li, Jingbo Yang, Yong Kong, Meizhen Gao, Journal of Magnetism and Magnetic Materials, Volume 172, Issues 1-2, 1 August 1997, Pages 165-172.

[16] A.V. dos Santos, C.A. Kuhnen, Solid State Communications, Volume 98, Issue 2, April 1996, Pages 123-128.

[17] Pollini et al, Physics Reports, Volume 355, 2001, Pages 1-72.

[18] Toth, L.E., Transition Metal Carbides and Nitrides. Academic Press, New York, 1971.

[19] Storms, E.K., the Refractory Carbides. Academic Press, New York, 1967.

[20] Ohring, M., The Materials Science of Thin Films.Academic Press, San Diego, 1992, Page 547.

[21] Bunshah, R.F., Deshpandey, C.V., Vacuum, Volume 39, 1989, Page 955.

[22] Kim, S.D., Jin, S.J., Hong, M.R., Kim, C.T., J. Electrochem. Soc. Volume144, 1997, Page 664.

[23] Wang, S.Q., Allen, L.H., J. Appl. Phys. Volume 79, 1996. Page 2446.

[24] I.R. Shein., A.L. Ivanovskii., Journal of Solid State Chemistry Volume 177, 2004, Pages 61-64.

[25] Z. Dridi., B. Bouhafs., P. Ruterana., H Aourag, J. Phys.: Condens. Matter, Volume 14, 2002, Pages 10237-10249.

[26] Tetsuya Takahashi., Denis Music., Jochen M. Schneider., Scripta Materialia, Volume 65, 2011, Pages 380-383.

[27] A.V. Gil Rebaza., J. Desimoni., E.L. Peltzer y Blanca., Physica B, Volume 407, 2012, Pages 3240-3243.

[28] A.V. dos Santos, J.C. Krause, C.A. Kuhnen., Physica B, Volume 382, 2006, Pages 290-299.

[29] R.S. de Figueiredo., C.A. Kuhnen., A.V. dos Santos, Journal of Magnetism and Magnetic Materials, Volume 173, 1997, Pages 141-154.

[30] C. Paduani., Journal of Magnetism and Magnetic Materials, Volume 278, 2004, Pages 231-236.

[31] C.A. Kuhnen., R.S. de Figueiredo., A.V. dos Santos, Journal of Magnetism and Magnetic Materials, Volume 219, 2000, Pages 58-68.

[32] A.V. dos Santos, Physica B, Volume 396, 2007, Pages 1-7.

[33] A.V. dos Santos, C.A. Kuhnen., Journal of Solid State Chemistry, Volume 182, 2009, Pages 31833187.

[34] C.A. Kuhnen., A.V. dos Santos, Journal of Magnetism and Magnetic Materials, Volume 130, 1994, Pages 353-362.

[35] C.A. Kuhnen., A.V. dos Santos, Solid State Communications, Volume 85, No. 3, 1993, Pages 273-279.

[36] H. L. Skriver, "The LMTO method", Springer Series of Solid Sciences, New York, 1984.

[37] O.K. Andersen, Phys. Rev. B, Volume 12, 1975, Page 3060.

[38] W.A. Harrison, Electronic Structure and the Properties of Solids (Freeman, San Francisco), 1980. 
[39] Brich F., J. Geophys. Res, Volume 83, 1978, Page 1257.

[40] F.D. Murnaghan, , Proc. Natl. Acad. Sci USA, Volume 30, 1944, Page 5390.

[41] von Barth U. and Hedin L., J. Phys. C, Volume 5, 1972, Page 1629. 